\title{
Hydraulic power plant machine dynamic diagnosis
}

\author{
Hans Günther Poll ${ }^{\mathrm{a}, *}$, José Carlos Zanutto ${ }^{\mathrm{b}}$ and Walter Ponge-Ferreira ${ }^{\mathrm{b}}$ \\ ${ }^{a}$ Voith Siemens Hydro Power Generation Ltda, São Paulo, Brazil \\ ${ }^{\mathrm{b}}$ Instituto de Pesquisas Tecnológicas de São Paulo-IPT, São Paulo, Brazil
}

\begin{abstract}
A method how to perform an entire structural and hydraulic diagnosis of prototype Francis power machines is presented and discussed in this report. Machine diagnosis of Francis units consists on a proper evaluation of acquired mechanical, thermal and hydraulic data obtained in different operating conditions of several rotary and non rotary machine components. Many different physical quantities of a Francis machine such as pressure, strains, vibration related data, water flow, air flow, position of regulating devices and displacements are measured in a synchronized way so that a relation of cause an effect can be developed for each operating condition and help one to understand all phenomena that are involved with such kind of machine. This amount of data needs to be adequately post processed in order to allow correct interpretation of the machine dynamics and finally these data must be compared with the expected calculated data not only to fine tuning the calculation methods but also to accomplish fully understanding of the influence of the water passages on such machines. The way how the power plant owner has to operate its Francis machines, many times also determined by a central dispatcher, has a high influence on the fatigue life time of the machine components. The diagnostic method presented in this report helps one to understand the importance of adequate operation to allow a low maintenance cost for the entire power plant. The method how to acquire these quantities is discussed in details together with the importance of correct sensor balancing, calibration and adequate correlation with the physical quantities. Typical results of the dynamic machine behavior, with adequate interpretation, obtained in recent measurement campaigns of some important hydraulic turbines were presented. The paper highlights the investigation focus of the hydraulic machine behavior and how to tailor the measurement strategy to accomplish all goals. Finally some typical recommendations based on the experience obtained on previous diagnostic reports of Francis turbines are performed in order to allow a better and safe operation of these power plant units.
\end{abstract}

Keywords: Diagnosis, dynamic measurement, von Karman, power, Francis, frequency, speed no load, strain, stress

\section{Introduction}

Francis hydraulic units are reaction machines that are designed according to specific hydraulic conditions limited by the hydrological variables such as head and the flow yearly variations. The hydro conditions of the power plant location will drive the Francis machine design and goals such as high efficiency unit operation and the maximum yearly unit utilization are pursued in order to increase the return of the investment.

This type of machine is designed according to computational fluid dynamics techniques and a scaled model is manufactured and tested on hydraulic laboratories to assure the design before the start of the real full scale machine prototype. The Hydraulic model test is also required because each machine is at the end tailored to specific hydro conditions that are rarely equal to any already existent one.

Although the turbine model test is suitable to understand the machine behavior at every loading condition from a hydraulic point of view, the full comprehension of the machine behavior and especially its structural response cannot be scaled up to prototype size according to the homology rules. The influence of the water intake inertia is

\footnotetext{
*Corresponding author. E-mail: hans.poll @vs-hydro.com.
} 


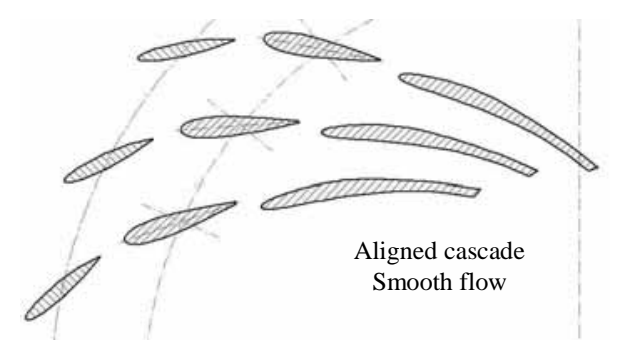

Fig. 1. Tamdem Cascade - best point.

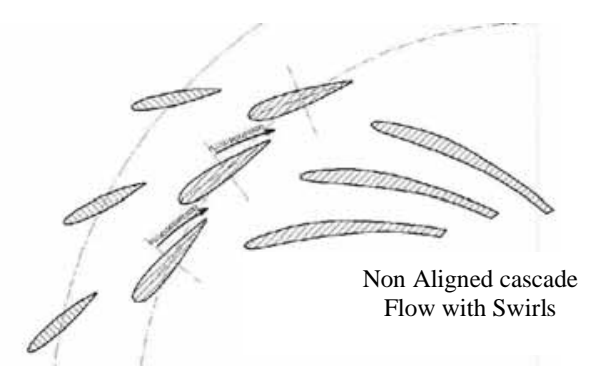

Fig. 2. Tamdem Cascade intermediate point.

something that can be checked only at prototype scale. There are some uncertainties that can be finally checked only at the prototype scale.

Added to this fact the structure mechanical response can hardly be evaluated on the model scale especially because the model runners are being manufactured in bronze, which is much different than prototype runners that are typically performed in martensitic stainless steel inclusively with welded on buckets. The response of the components that are under water such as the stay vanes and the runner is particularly important because the flow will induce vibrations on those components and can be intense if adequate measures are not performed.

Although one of the main design focus considers the goal of low dynamic stresses and low induced vibration, the nature of a fixed runner blade machine, such as Francis machines, will get some operating conditions suitable to rough dynamic loads and long term operation on that particular region might reduce the component life time at the highest stressed areas.

Due to the fact that Francis machines contain fixed blades, different from Kaplan units where another degree of freedom is added by the blade rotation that optimizes the blade inflow geometry according to the flow behavior, here the inflow angles are fixed and designed for some particular flow conditions. For intermediate load conditions the flow might get unstable. For a fixed head, the power changes according to changes in flow discharge that is regulated by the distributor wicket gate position that is, ultimately, driven by the electronic governor system. In order to establish the flow discharge the wicket gates rotate from an aligned position to a closed position. Since a Francis runner is typically a welded construction where the blades are attached to a crown and band, one unique blade geometry is defined for all operating loads even for a set of operating heads. This geometry is tailored to the rated or best efficiency region that is limited according to the design. By analyzing the tandem cascade that includes the stay vane, wicket gate and blade profile of a runner one can recognize that for one specific opening the water through the cascade will flow smoothly, "Fig. 1". By closing the distributor the wicket gate blocks the flow and it is also possible to see that the flow streamline will change significantly from the best operating point condition, "Fig. 2". This wicket gate intermediate position decreases the flow by incrementing the head loss but also generates flow swirls that pass through the runner inducing dynamic loads to the structure. The knowledge of amplitudes and frequencies of such dynamic loads is one of the goals of the proposed diagnosis. "Figures 3 and 4 " show two sequences of the real flow behavior inside a model runner.

The time to have a fatigue crack being initiated at the high stressed areas of a runner, for instance, is fully dependent of how the energy company or dispatcher operates the machine. If the operating condition considers the 


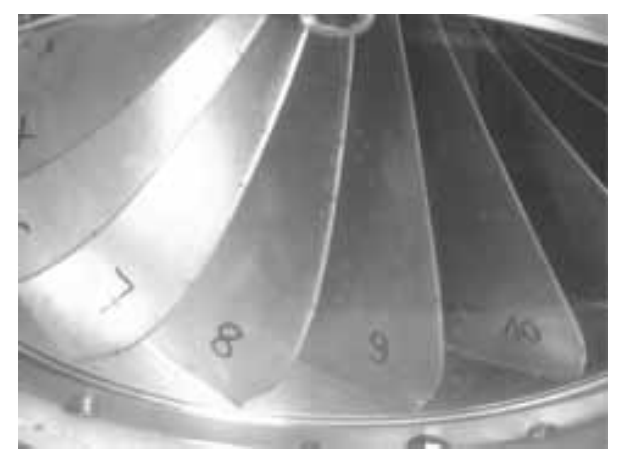

Fig. 3. Flow in the Runner-Best point.

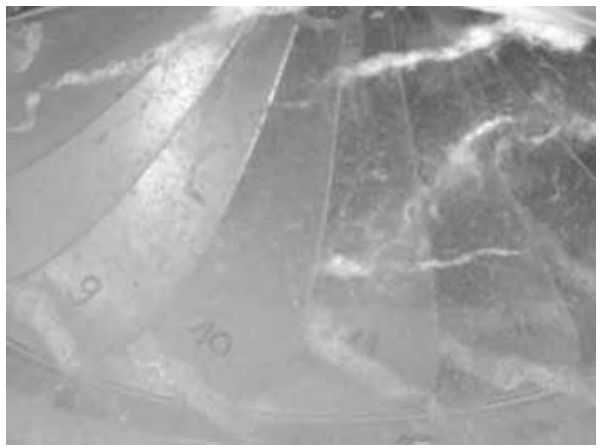

Fig. 4. Flow in the Runner-Lower opening.

neighborhood of the rated machine point without many starts and stops the runner will probably have its maintenance costs driven to the lowest value. However if the machine is often operated at speed no load or any rough unstable condition with many starts and stops the chance to have cracks being initiated and to have considerable maintenance costs will be high.

The machine full diagnosis as described here will practically eliminate all prototype unknown variables and will provide the full comprehension of the machine behavior at every load condition. The generated data will allow one to get all fatigue related safety margins for each operating condition and shall allow the owner to optimize the operating conditions to reduce the possible change for high maintenance runner costs.

Occurrences of possible severe high frequency exciting phenomena will be discovered during the machine diagnosis and the cause will need to be eliminated in order to preserve the machine lifetime. Nevertheless occurrences of low frequency exciting loadings must be investigated in order to define and especially fine tune the most optimum way to run the machine combining hydraulic and mechanical performances.

It is also very common in several countries such as Brazil and China to have the machine operating conditions being defined by a dispatcher agency. For that cases it is particularly important to conduct the prototype diagnosis and influence the operating definitions from a structural mechanics and maintenance point of view.

It is also important to mention that during the diagnosis the air injection or natural air admission can be measured and tested. Air admission can smooth the unstable operating points and this evaluation, although predicted during the model test, must be tailored during prototype test where cause and effect are adequately correlated.

The Technological Research Institute of the State of Sao Paulo (IPT), a 105 years old non-profit institution owned by the of the state government, performed innumerous hydro machine diagnosis in tight collaboration with energy companies and the hydro machinery industry. The diagnosis experience of IPT is supported by over 15 measured Francis runners and stay vanes conducted in units from $47 \mathrm{MW}$ up to over $700 \mathrm{MW}$ since 1978. Voith Siemens (VSH) is a joint venture full liner company of Voith (137 years old) and Siemens (157 years old) that designs, manufactures, installs and operates hydro units. VSH uses to work in collaboration with measurement specialists 


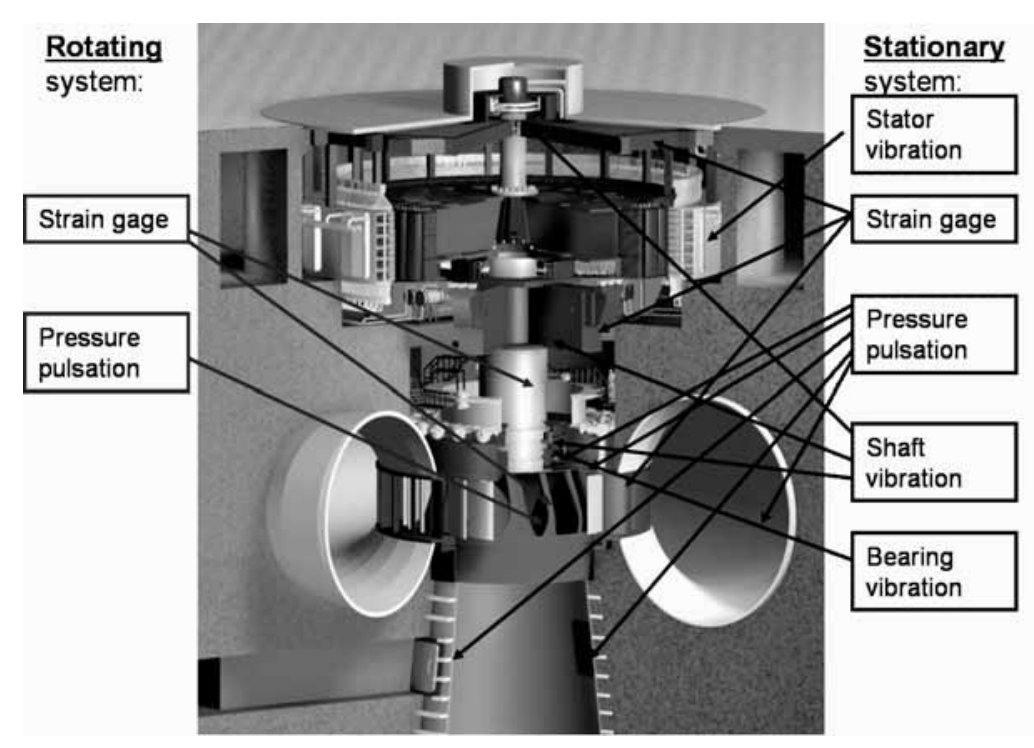

Fig. 5. Location of Sensors on Francis unit.

such as the Naval and Oceanic group of IPT and also performed measurements by itself on Francis machines of different sizes, always focusing on findings that increase the reliability of the hydro unit.

A method to acquire correct information and how to get and find out important conclusions from the collected data is presented. Also some recommendations based on conclusions taken from several machines investigated around the world are presented at the end.

\section{Measurement techniques}

The instrumentation system consists of data acquisition units, which have their own IP addresses and communicate with a notebook using Internet protocol. The system allows simultaneous measurement of up to 256 channels with more than 1000 samples/second per sensor if all sensors are used. If the number of channels decreases it is possible to increase the sampling rate (90 channels with 4096 samples/second per sensor).

The measurement strategy involves strain gages that are submerged in water, pressure transducers, mini waterproof pressure transducers, accelerometers and approximation meters. It is very important to measure all quantities with adequate equipment that synchronizes all data and so that the physical information can be correctly calibrated to the acquired data. It is also clear that an entire hydro machine diagnosis requires that all these measurements must be acquired at the same time in order to allow correct correlation of measurement data with the physical data. "Figure 5" shows the typical location of the measurement sensors installed in the power unit.

Waterproof rosettes and unidirectional strain-gages are used for measuring strain on the runner and torsion strain of the turbine shaft (torque). These kind of strain-gages are attached to the runner blades, submerged in water, and the wires that come out from each strain gage are connected to signal conditioners. Strain gages and cables are totally covered with a special resin that provides mechanical and electrical isolation.

Since Francis runners are subject to high loads it is necessary to search the correct position of each strain-gage to be attached to runner blades. Typically the stress distribution of a Francis runner has a high gradient shape and it is necessary to know the mechanical structure in order to select the correct location for each strain gage. A remark must be made: every runner has a different stress field and this is related to the blade geometry and its particularities. With the evolution of computational fluid dynamics in the last decades several new geometries were developed mainly focusing higher efficiencies inclusively at part loads. This means that the prototype measurement campaign must be tailored according to the power unit conditions and especially according to the runner. The stress analysis must also be conducted in several loading conditions in order to search adequately the highest stressed points and attach 

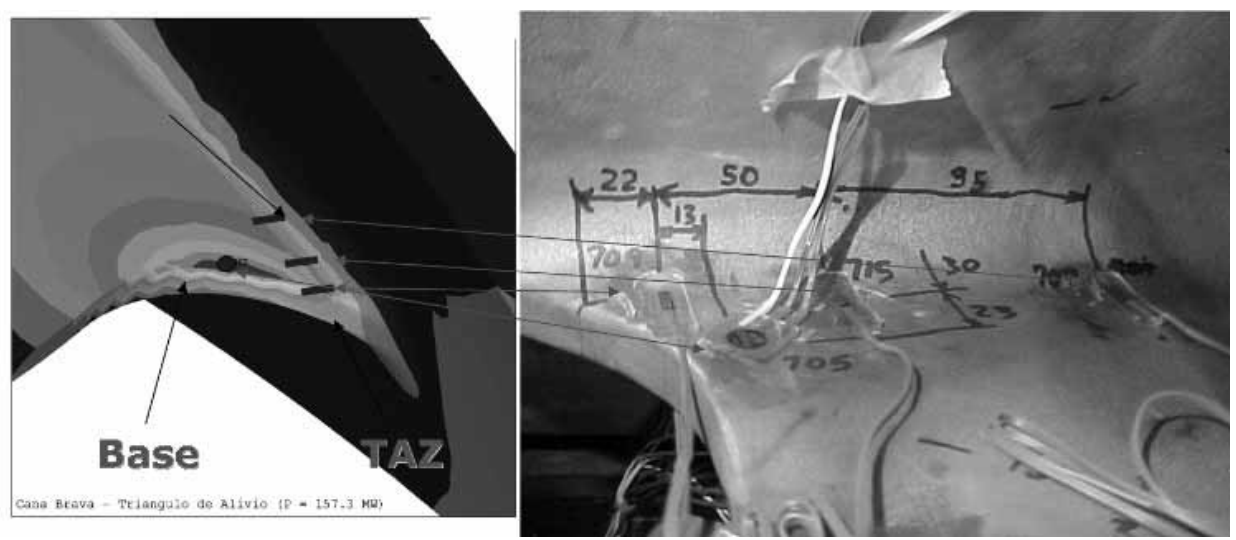

Fig. 6. Strain Gages guided by structural analysis.

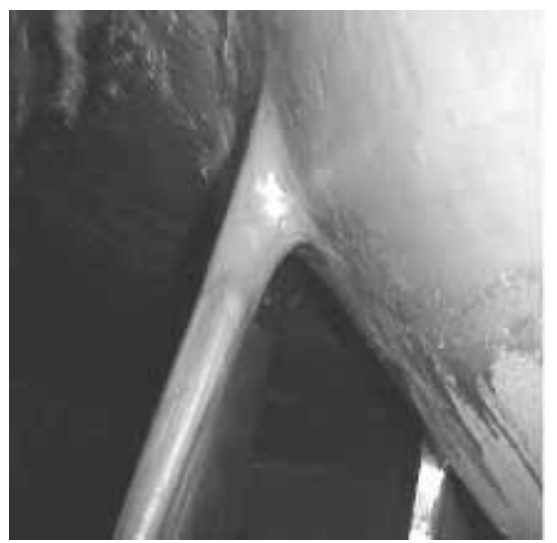

Fig. 7. Resin Putty protection at the discharge egde of the blade to crown.

on that location the strain gages. "Figure 6" shows the correlation between the results of the structural analysis and the position of the strain gages. The strain gages are place where high stress gradient occurs . It is important also to consider the direction of the stress in order to acquire most representative signals. "Figure 7" shows the resin putty protection that covers the strain gages and the cables. These elements will operate under water and the resin must be reliable for at least 10 hours of operation under several different conditions. Modal analysis data must also be used in order to specify the most interesting position for the strain gage. The current calculation methods are accurate enough to predict within mm the stress location and this accuracy is highly dependent of the calculated flow and pressure distribution that is computed on CFD systems. This information needs to be cross checked with metallurgical data especially at the welded seams. Once the stress field is know the welding seam regions such as the transition area and the thermal affected zone (TAZ) must be identified in order to precisely guide the introduction of the strain gages. "Figure 8" shows also the position of unidirectional strain gages at the entrance of a Francis rotor and its correlation with the stress field. "Figure 9" shows the results of modal analysis and stress amplification areas guiding where to place the strain gages.

Pressure transducers are used to measure pressure (spiral case, head cover, draft tube, crown) and accelerometers to measure acceleration and vibration (band, crown, bearings). Mini pressure transducers can be also used for local under water dynamic acquisition. Power, speed and wicket gate opening (governor) are also simultaneously measured. All pressure transducers and proximeters must be calibrated before starting measurement. Their static outputs need to be also correlated with the current head level or distances for instance in order to guarantee that the quantities can be compared to reference values adequately. 


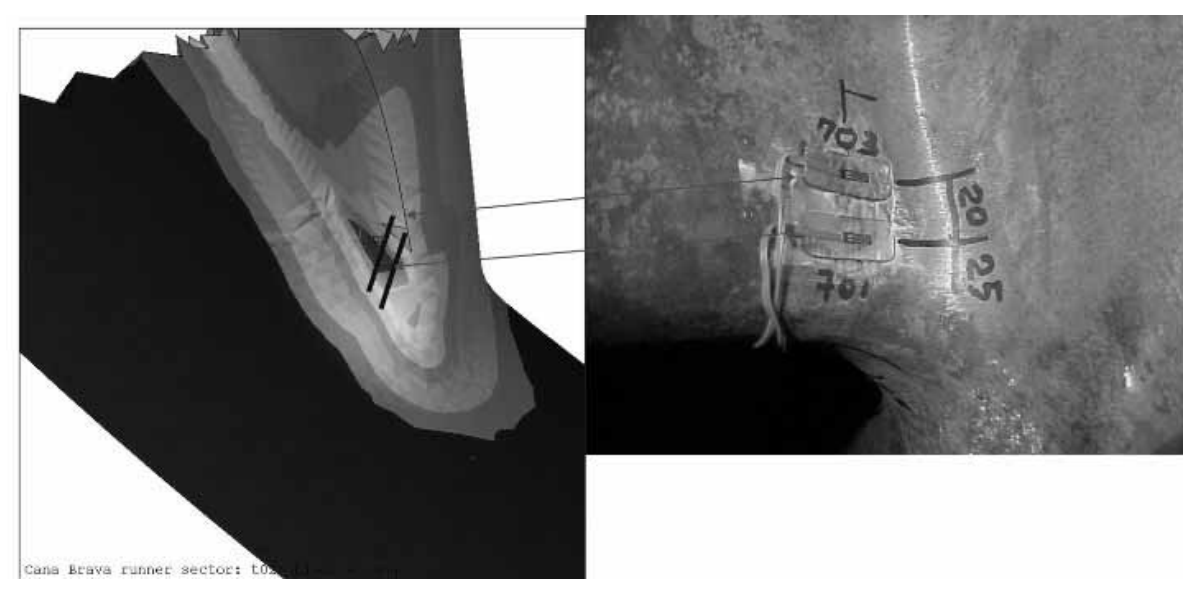

Fig. 8. Strain Gages for runner entrance edge.

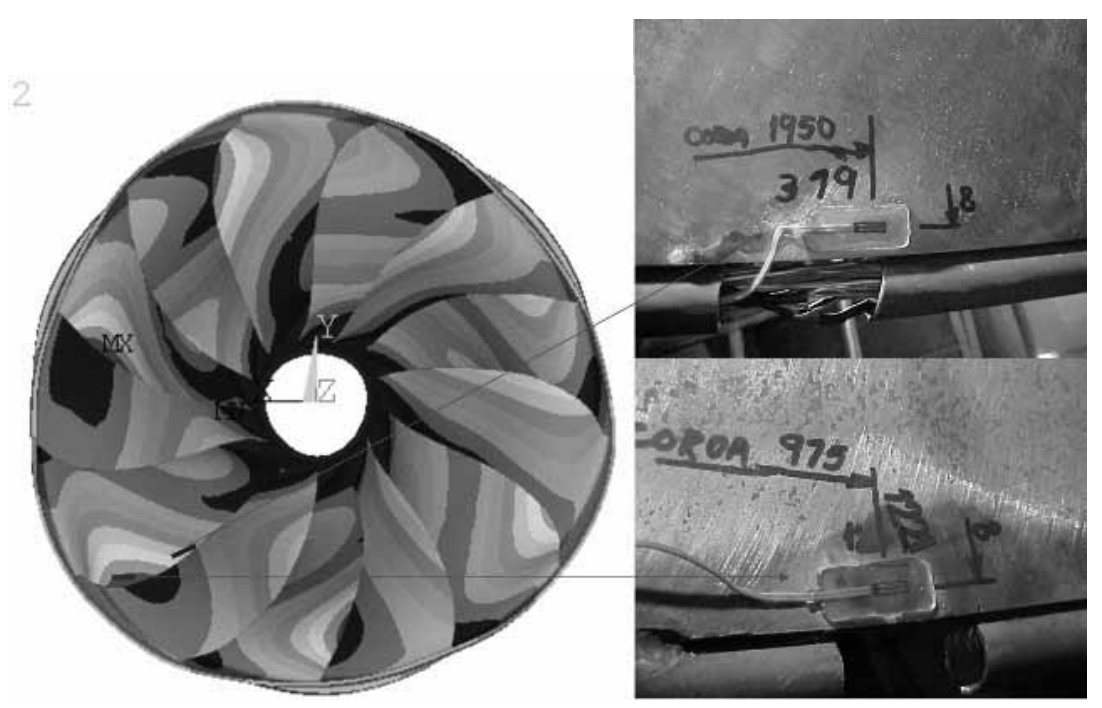

Fig. 9. Strain gages for mode shape investigation.

In many examples the machine diagnosis as described here is combined with the relative efficiency test generally called "index test". Here the flow is also measured by the acquisition of pressure in two different locations of the inlet pipe of the spiral case by the use of a Winter Kenedy sensor. This differential pressure that is being measured can be correlated with measured flow obtained during the model test in the laboratory and discharge is obtained.

The machine diagnosis is then suitable for the determination of relative efficiency values. Natural airflow, typically through the shaft can be also measured by an anemometer directly of at least by the acquisition of air gap opening of the aerating valve. This data will provide additional important information for the machine stability investigation.

The signal conditioners (for the strain-gages, accelerometers and pressure transducers on blades) are plugged inside control units installed on top of the generator shaft and rotate together with the runner, acquiring data, "Fig. 10".

Other control units, not rotating with the runner, are used for measuring power, speed, opening, pressure, shaft oscillation, strain on stay vanes and any other sensor placed outside the turbine, "Fig. 11".

One of the control units called "master" sends a synchronization signal via cable to other control units on top of the shaft and, via "slip-ring", to the external control unit, so that all signals are simultaneously sampled, in every measurement. The measured signals outputted by each control unit are sent to a "hub" and from it to the "PC Card" of a notebook, where all signals are stored and real time displayed both in time and frequency domain. 


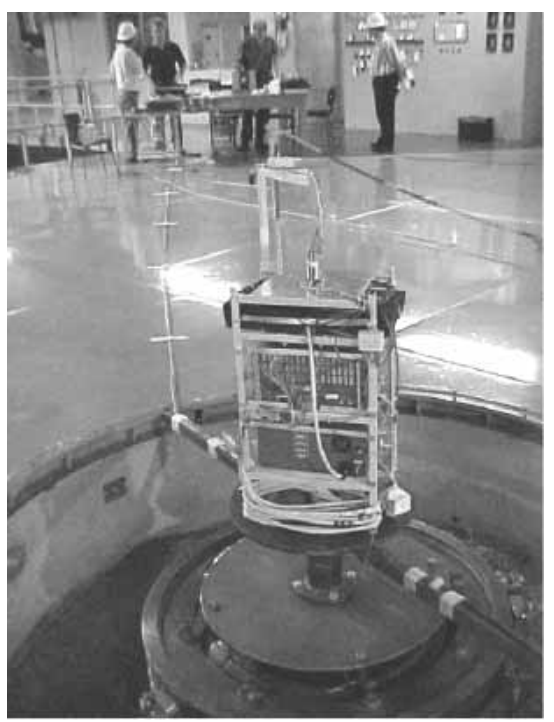

Fig. 10. Measurement Control unit.

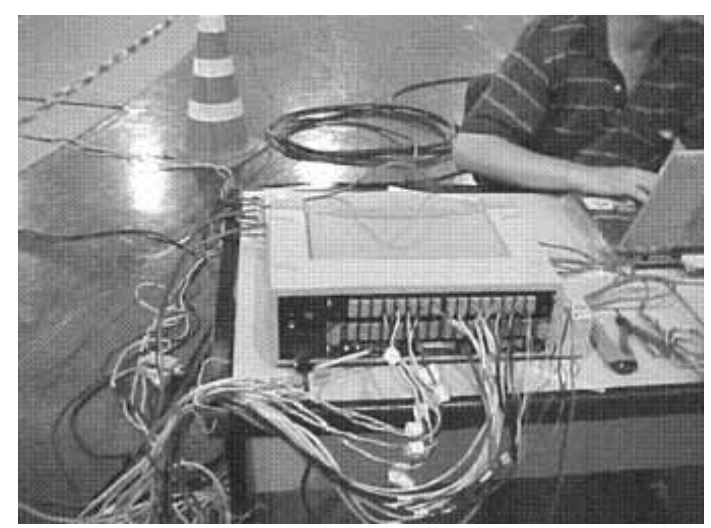

Fig. 11. Non rotating sensors conditioners.

Each sensor from the runner is connected to an individual signal conditioner (each strain-gage is connected via shielded twisted conductors, using the three-wire method), where the signals are automatically balanced, amplified, calibrated and low-pass filtered (anti-aliasing). The cut-off frequency depends on the signal bandwidth of interest, which is tailored for each measured machine according to the design conditions.

From the conditioners the signals are sent to an analog to digital converter, where they are simultaneously sampled (1024 to 4096 samples per second, per sensor), multiplexed, converted to digital serial form (with 16 bits resolution), then formatted and finally sent, via slip-ring to a "hub" and then to a notebook, to be displayed and stored in data files. Signals from the sensors outside the turbine are filtered, sampled and converted simultaneously with the sensors on the runner and sent to the notebook via cable through the same "hub".

During measurements signals are real time displayed on the screen both in time and frequency domain, side by side. They may be organized in groups of channels to be displayed together and with a simple mouse click it is very easy to change the set of channels displayed on the screen.

Before starting measurement, the strain-gages are balanced and calibrated with the runner stopped and then recorded as the reference ("zero"). 


\subsection{Measurement campaign method}

Due to the fact that some strain gages, pressure transducers or even accelerometers will acquire data submerged in water and although every putty protection still has some limitations it is very important to plan adequately the measurement sequences so that all objectives are fulfilled in the first 2 measurement hours.

Typical prototype measurement goals are related to the determination of following quantities:

- Mean stress at runner blades and stay vanes at steady state operating conditions.

- Low frequency pressure pulsations at the draft tube and its effect on unit stability.

- Characteristic stress amplitudes of high stressed areas at runner blades and vanes.

- Dominant frequencies on rotating and non rotating parts with special focus on high frequency phenomena.

- Stresses and pressure pulsations developed during starting up and stopping transients.

- Dynamic stresses developed under speed no load conditions.

- Effect of different wicket gate opening periods on stresses and pressure pulsations for transients.

- Effects of air injection or natural air admission on every steady state for machine dynamics.

- Correlation of unit vibrations with possible causes such as pressure pulsations or other phenomena.

Steady state conditions and transient conditions need to be investigated. For the measurements the governor must be put in constant opening mode because it is necessary to make sure that the wicket gates operate in fixed position and this will keep same hydraulic flow conditions. The first part of the test consists to start the machine up to nominal rotation without synchronization, maintain this condition for few minutes and stop the machine. After this first step all sensors must be reviewed and the acquired data must be analyzed. The second step is to start the machine, synchronize it and increase power up to maximum power, maintain for few minutes and decrease the power step by step. The steps at this time must be selected based on the machine hydraulics. The machine opening should be kept constant at each step for 3 minutes in order to allow adequate FFT computation for frequency domain analysis.

After this second phase the entire strain scenario is evaluated and a dominant frequency analysis might be performed at the most important steady states. Now, deep investigation can be performed by selecting certain operating regions maintain the machine at these specific points for some minutes. At the end a load rejection of full load is recommended for investigation. At the end of the measurements, with the runner stopped, the reference signal must be recorded again.

Results are presented as time series graphs, tables and graphs of frequency spectra, waterfall graphs, average, characteristic amplitude, rms, standard deviation, maximum and minimum values. Results from strain-gages may also be expressed in stress. For example, principal stress and equivalent stress versus time (dynamic) can be computed using the instantaneous data measured. Frequency spectra and graphs of the stresses may then be obtained.

\section{Experimental modal analysis}

One of the most important aspects of machine diagnosis is the fact that the natural shape modes of the mechanical components are not being excited especially to what concerns the wicket gates, stay vanes and the turbine runner. For that occurrence it is necessary to have a coincidence between exciting and natural frequencies and in order to anticipate eventual problems an experimental modal analysis can be recommended. The numerical modal analysis must be performed previously because these frequency and mode shape results will guide the experiment and it is through this experiment that the frequencies will be finally precisely determinated. The results of the experiment will also be used as a feedback for the calculation method of the component embebed in water.

On the experiment, the structural components of the turbine are being excited at several points with a hammer that is equipmed with an accelerometer. The structure response, its vibration, is also recorded by the used of accelerometers that are maintained connected to the component. After signal amplification and adequate filtering the excitation signals and responses are recorded by a digital acquisition system. The frequency response fuctions (FRFs) are estimated then by the use of FFTs (Fast Fourier Transformation) for each pair of excited and response data points. The FRFs represent then the dynamic behavior of the structure, and permit to identify the resonance frequencies of each component as one can see at "Fig. 13". The coherent fuction also is used to evaluate the quality of 


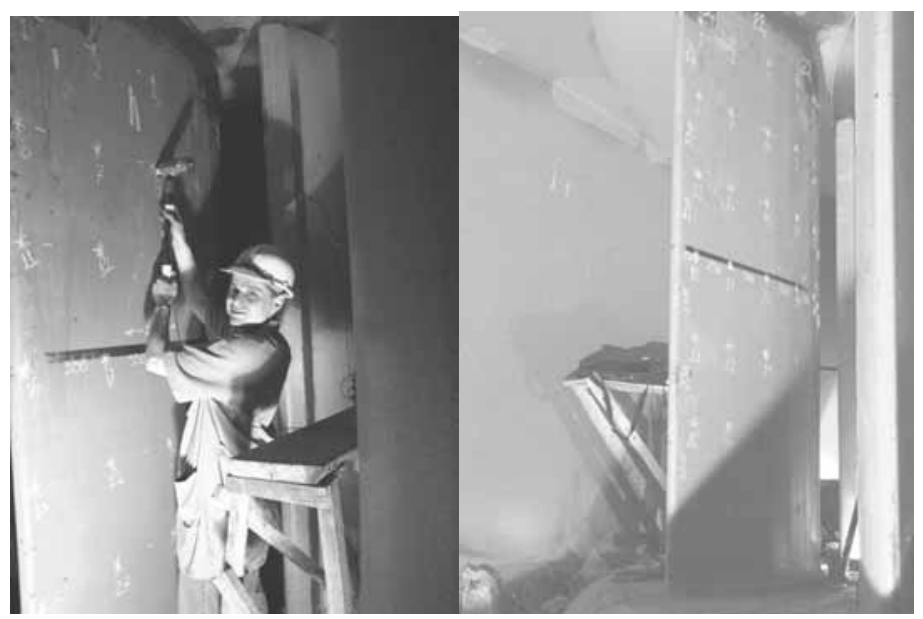

Fig. 12. Mesh and experimental modal analysis technique.

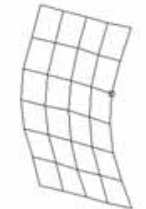

$1^{\text {st }}$ Mode

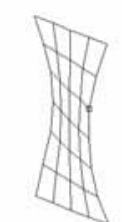

$2^{\text {nd }}$ Mode

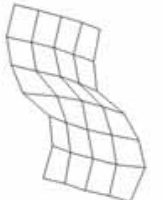

$3^{\text {rd }}$ Mode

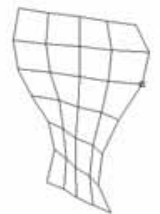

$4^{\text {th }}$ Mode

Fig. 13. Typical natural vibration modes for stay vanes.

the acquired signal and experimental erros that might occur due to noise problems. Then after the modal parameters are identified through function adjustments [4]. Normaly methods on the frequency domain are used [6] when suscessive measurements of FRFs are used for each pair of points. Typically, for large Francis runners (D $>6 \mathrm{~m}$ ), the frequency range goes up to $500 \mathrm{~Hz}$ and it is determined by the maximum possible exciting frequency associated with von Karman trailing shedding.

All relevant points for the adequate measurement of the FRFs are selected in such a way that all vibration modes of the structural components can be clearly identified. Due to access difficulties exausting measuring meshes are to be avoided and focus must be put on the relevant regions especially for the dischage region of runner buckets. On Stay vanes a regular mesh can be easily designed and this allows to distinguish torsional and flexional modes. For this kind of component, that allow reasonable access, see "Fig. 12", and are also not damped, it is possible to measure a larger number of points and this allows a better vizualization of the shape modes as shown on "Fig. 13".

The mode shape behavior of each runner bucket can be characterized by measuring only the discharge edge of the bucket. "Figure 14" shows typical results. If a larger mearurement mesh for the bucket is applied the shape modes as shown on "Fig. 15" can be also drawn.

For the runner it is important to use a measurement point approximately in the center of the discharge edge of the bucket in order to coupled the movement of adjacent bucket and use this data to build the entire runner mode shape [6, 7]. The coupled movement of the runner buckets can be represented at "Fig. 16". Here the transversal displacement of each bucket is shown in radial direction. In the first mode shape, $n=0$, all buckets got movements in the same direction. On the second mode shape, $n=1$, half of the buckets move in a counter phase to the remaining buckets. In the third mode shape, $n=2$, approximately one quarter the buckets move in opposite phase to the next quarter in a similar way of the further shape modes where more buckets are then moving in opposite phases in respect to the adjacent buckets.

This method allows the determination of the characteristics of the vibration modes and this indicates the frequencies that are more susceptive for resonance. The number that shows this tendency for cyclic periodic structures such as 


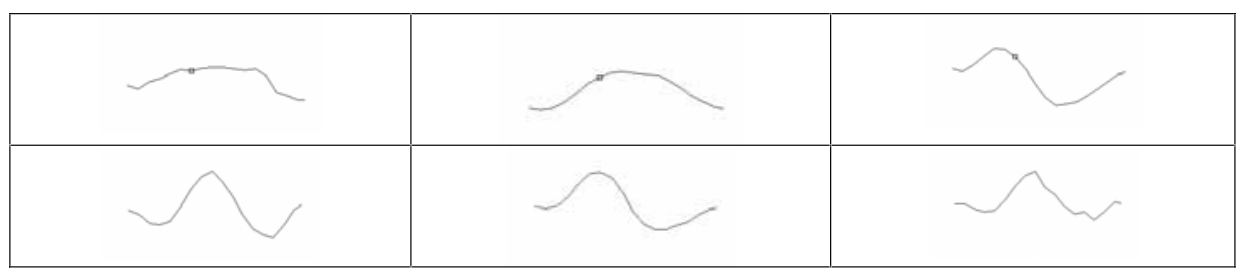

Fig. 14. Typical natural vibration modes for the discharge edge of buckets.

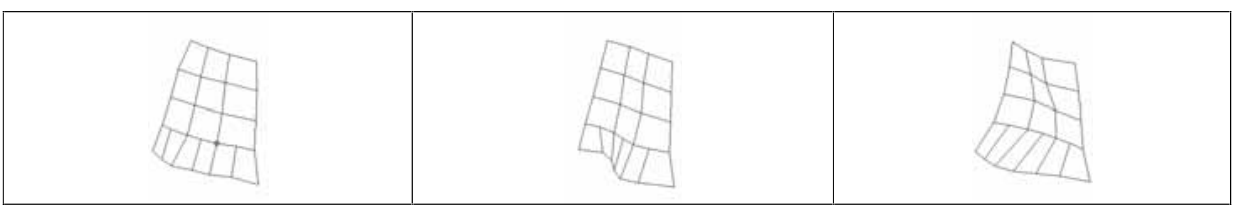

Fig. 15. Typical natural vibration modes for runner buckets.

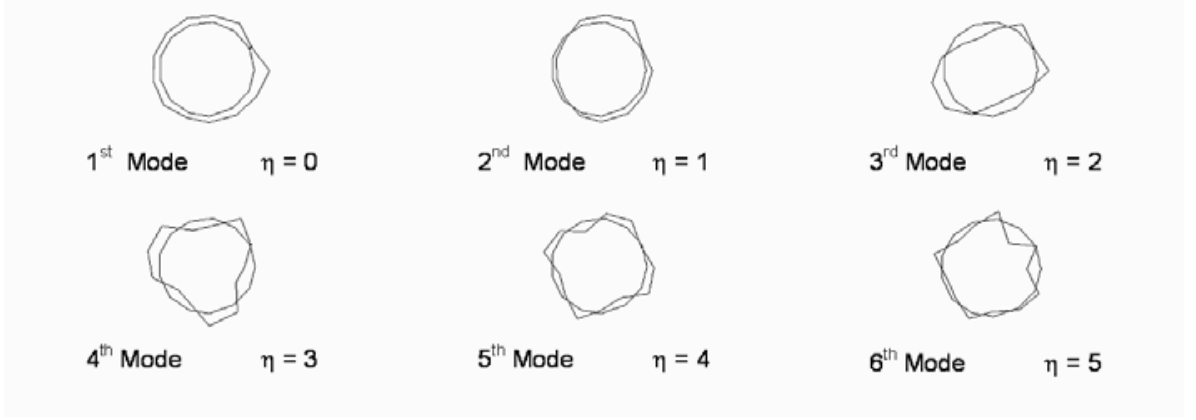

Fig. 16. Natural vibration modes for the francis runner.

a runner is the number of nodal diameters or more precisely the wave number. This number indicates the integral wave number that one natural vibration mode contains along the runner circumference. These numbers associated to the correspondent natural frequencies represent the main dynamic aspects of the runner [10]. "Figure 17" shows the comparison of experimental data and calculated data. In this chart the natural frequencies are plotted in function of the diameter nodes of each mode shape. It is a special characteristic of cyclic periodic structures to present several natural frequencies in a small frequency range and this can be observed on "Fig. 18".

The modal calculation of runners is a very important step in the design of such components. The calculation is also typically performed in two steps where the first one considers surrounding air and the second considers surrounding water. This last calculation must be done by the use of fluid elements and typically the obtained frequencies are lower then the ones calculated in air due to the damping that is caused by the water. However each mode shape has a different damping reduction factor when the results in air and in water are compared. The experimental modal analysis, due to limitations of safety and size, can be done also only in air and this evaluation is important to get the final responses of the manufactured component. Results such as the ones presented on "Fig. 17" validate the calculated data in air and support the results of the calculation that was done in water.

The fact that such structures may present many frequencies and mode shapes concentrated in a small frequency range requires accurate prediction of the calculated data and of the possible exciting frequencies so that any possible resonance can be totally avoided.

\section{Typical machine stability analysis}

Due to the fact that the method allows one to observe all sensors during machine operation in real time some conclusions can be taken right after the test. High and low exciting frequencies can be verified during the test and the 


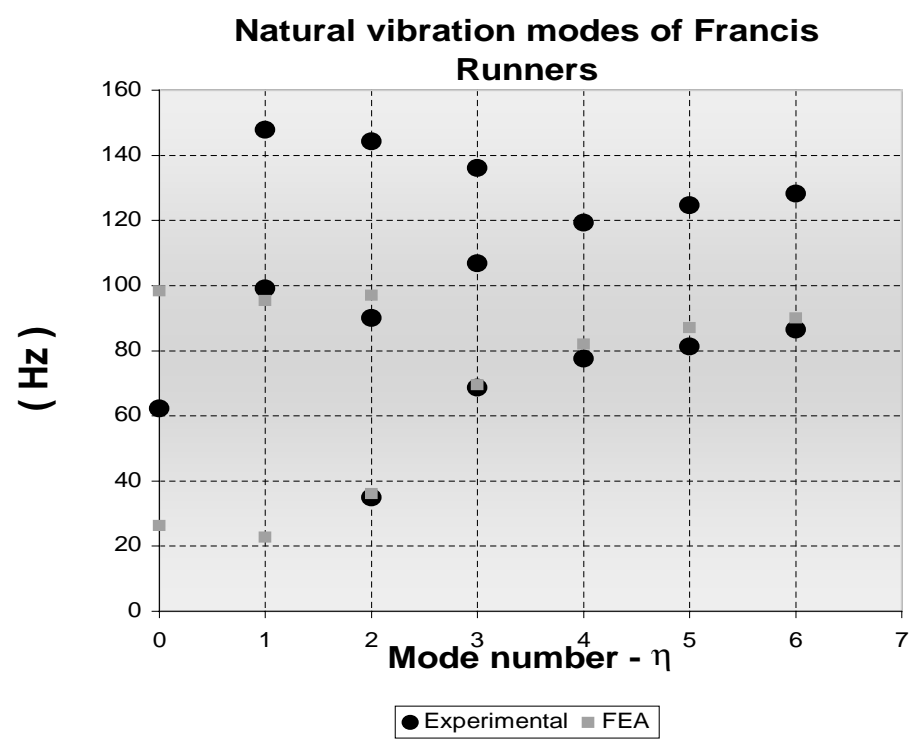

Fig. 17. Experimental x calculated results.
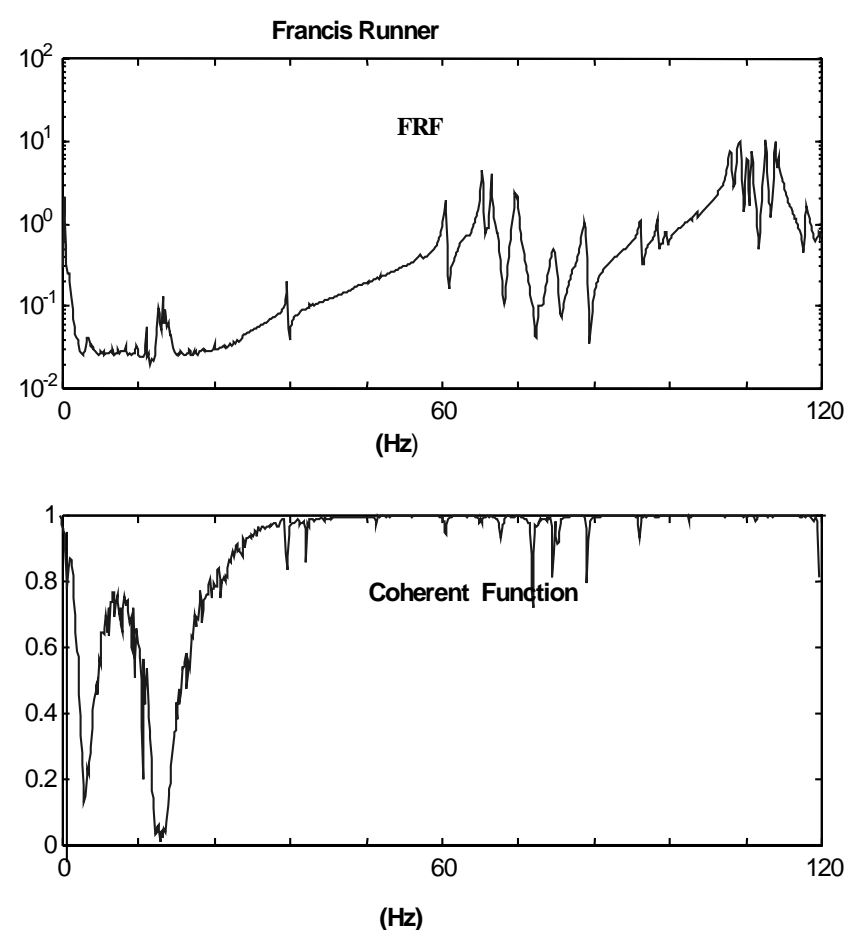

Fig. 18. Typical FRF for Francis Runner.

relative efficiency results could also be forecast with good accuracy during the test using the mean observed values of the differential pressure transducer. However one important observation is the adequate set up of the sampling rate and the filter cutoff frequency. For that purpose the following excitation frequencies should be considered: Wicket gate passing frequency up to the 3rd harmonics, electrical frequency, vortex rope frequency (Rheinghans), machine runaway, over speed frequency and the possible von Karman excitation frequencies. 


\begin{tabular}{|c|c|c|c|c|c|}
\hline $\begin{array}{l}\text { Power } \\
\text { Plant }\end{array}$ & $\begin{array}{l}\text { Discharge } \\
\text { edge }\end{array}$ & $\begin{array}{l}\text { Strouhal } \\
\text { Number }\end{array}$ & $\stackrel{\mathrm{L}}{[\mathrm{mm}]}$ & $\begin{array}{c}\mathrm{b} \\
{[\mathrm{mm}]}\end{array}$ & $\begin{array}{c}\mathrm{h} \\
{[\mathrm{mm}]}\end{array}$ \\
\hline São Simẫo & & 0,28 & 2035 & 840 & 100 \\
\hline Água Vermelha & & 0,24 & 2215 & 777 & 89 \\
\hline Illha Solteira & & 0,26 & 2140 & 755 & 90 \\
\hline Marimbondo & 7 & 0,22 & 1830 & 730 & 80 \\
\hline Itumbiara & & 0,2 & 2130 & 954 & 140 \\
\hline
\end{tabular}

The observation limits will be set up by the filter cutoff frequency and if the occurrence of high frequency phenomena is going to be investigated the von Karman frequencies defined by Eq. (1) must be considered for the cutoff frequency set up. The von Karman frequency is calculated by Eq. (1) from the Strouhal number, the local flow velocity in $\mathrm{m} / \mathrm{s}$ and the runner blade outlet thickness. Typically the computation of the possible von Karman excitation frequencies must be done for every load condition and for several areas along the blade discharge edge. These frequencies need then to be compared with the runner natural frequencies in water in order to evaluate the chances to have a natural mode shape being excited by any von Karman frequency. Equation (1) shows the relation for the frequency.

$$
f=S_{r} \frac{\nu}{d}
$$

where $S_{r}=0.2-0.24$. $f$ is the vortex emission frequency, $\nu$ is the flow velocity at the separation point, and $d$ is the vortex shedding width at the separation point, near to the discharge edge.

The Strouhal number should be independent from the geometry and from the Reynolds value, however it is difficult to define that number as an unique constant.

Experimental tests conducted with special spheres and cylinders have demonstrated that the Strouhal number can be considered between 0.16 a 0.19 . The flow conditions around this bodies are more simple to be determined as the flow conditions around a plane body with a define geometry at the discharge edge. In 1952, Gongwer [12] proposed to add the boundary layer thickness to the geometry as main parameter for the described equation. Chen e Florjancic [13] tried to obtain a constant strouhal number, however when the boundary layer thickness was also considered the values start to vary between 0.18 and 0.19.For geometries typical used on hydraulic machines Liess [14] obtained Strouhal numbers by sets of several experimental data creating a reference list. The obtained results for 5 Brazilian Power plants are shown on "Table 1".

Miyagawa [15] compared experimental results on stay vanes with numerical results by bi and tri dimensional models and also shown that the Strouhal number varies from 0.2 to 0.24 according to the type of the discharge edge where the separation point defines the distance $\mathrm{d}$ to be considered as geometry thickness. The results obtained on stay vanes can be considered for the analysis of buckets, however here flow complexity and geometry variation add an extra difficulty and this is one of the motivations of the proposed method.

"Figure 19" shows result example of a Francis machine. These time series are related to a machine start and stop excursion inclusively with a full load rejection and came from static pressure transducers, positioning sensors and strain gages. This data is presented on a relative scale and do not show absolute values. The first curve from top to bottom shows the power variation, the second the speed variation and the third one the wicket gate opening values acquired with a special positioning sensor. These three curves are the reference data for the investigation of pressure and strain variation along the entire test excursion. The last three curves show respectively the pressure variation at the draft tube near to the inspection door, the strains measured at one blade near to its welding seam at the crown and the strains measured on a stay vane at the pressure trailing edge side. The excursion permits to get the total variation of the measured quantities and shortly understand the machine behavior along the power ramp. For instance, the pressure draft tube curve has on this time series high amplitudes up to $200 \mathrm{MW}$ of power, and has lower amplitudes when power increases to maximum getting again very intense dynamic portions during and after load rejection when the machine is hydraulically back to same condition as the starting one. This behavior typically transmits the vortex 


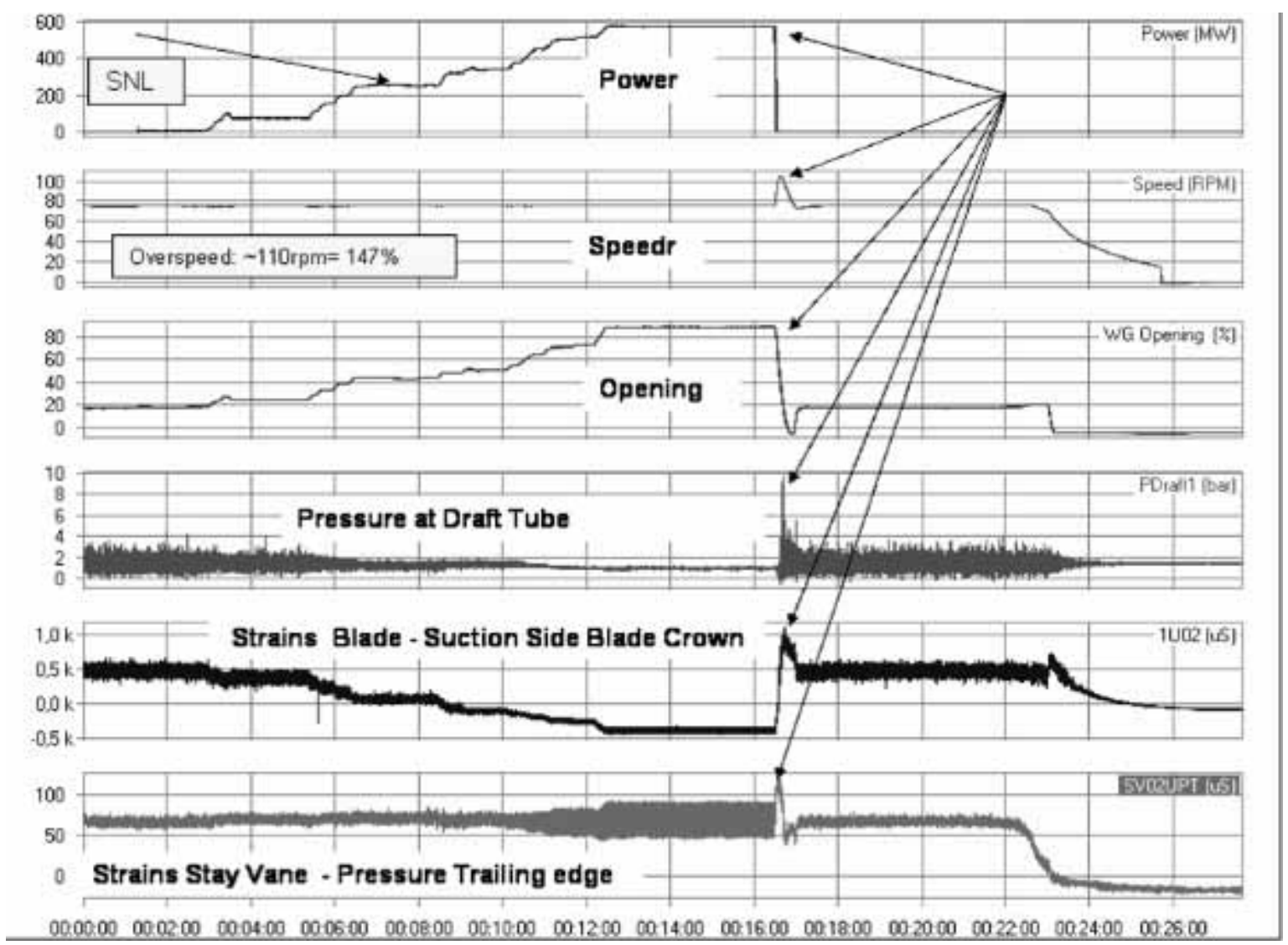

Fig. 19. Power Ramp on a Francis Machine.

rope behavior in the draft tube showing considerable pressure fluctuations during low loads. An instantaneous peak can be observed during load rejection, an instantaneous rapid effect of shock waves generated at rapid transients.

The strain curve of the blade shows compression higher than $100 \mathrm{MPa}$ during the initial operating phase, called "speed no load " (SNL) and a variation in tension direction as the power increases. At maximum power this strain gage shows approximately $100 \mathrm{MPa}$ of tension stress. During the load rejection the blade experiments a transient stress fluctuation that is over $200 \mathrm{MPa}$ coming back to the compression state and slowing down to zero when the machine stop as it would be expected. This curve shows two main subjects. There is a high frequency dynamic strain amplitude variation that is also larger during the same period when the pressure in the draft tube gets rough, smoothing out as soon the machine gets to maximum power. And there is a low frequency amplitude variation from start to stop that made the blade experienced a total compression to tension excursion. A fatigue calculation based on that curve can show if the low frequency variation is more dangerous to the runner blade material or if the high frequency variation is the one that causes more damage. On this particular case machine start and stop cycles are more critical to the blade material since the high frequency amplitudes are small and even this frequency value is not critical too.

The last curve shows the strain variation at the stay vanes where little higher dynamic amplitudes were observed during the period when the machine stayed at maximum power. Here an influence of higher velocities on larger openings increased the vibration at the stay vanes. Differently from the blades no compression-tension low frequency cycle was observed. However frequency analysis must also be conducted in order to investigate the fatigue accumulated damages to the material.

Machine stability can also be cross checked with the observations of "Fig. 12" when the data of the accelerometers and approximation sensors that are connected to the guide bearings are plotted. Low frequency pressure pulsations contain a higher amount of energy sufficient to influence the overall machine behavior. The high frequency 


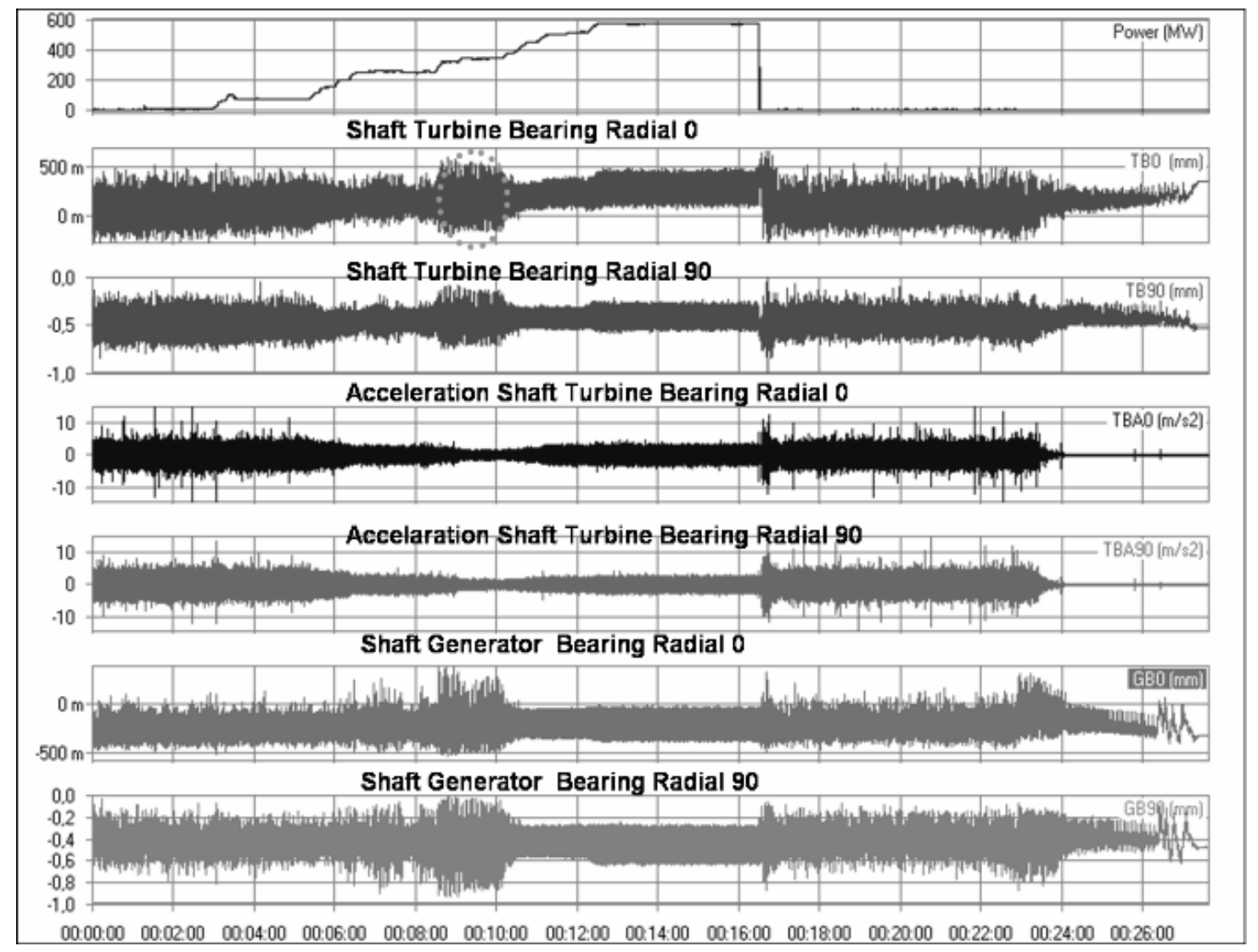

Fig. 20. Shaft vibration during power excursion.

phenomena are more often related to local problems and local material damages while the low frequency phenomena are felt on the entire machine. "Figure 20" shows the shaft relative and absolute vibration in radial and axial direction measured at the turbine and generator guide bearing of the same example used at "Fig. 12". The first curve is the reference power variation, the second and the third ones are the shaft relative bearing displacements in radial direction at 0 and 90 degrees. The fourth curve shows the axial vibration of the unit and the fifth the radial vibration still measured at the turbine bearing. The two last curves show the shaft displacements at the generator bearing. During SNL the unit behavior is rough with radial displacements almost fluctuating through the entire bearing gap, which probably increases its oil temperature. On this particular example, with power increase up to $250 \mathrm{MW}$ the machine gets smoother, however relative shaft displacements get higher again near to 300-350 MW but the absolute vibration continues getting smaller. Here the shaft orbit goes to a precession shape. Above that region the machine gets again very stable even considering the shaft displacements. At the load rejection transient high vibrations and displacements are noticed getting to the SNL condition. After unit shut down, in this particular example, no amplitude was recorded.

At other examples as shown in [1], the machine passed a high vibration situation during the stopping sequence due to resonance with high frequency phenomena. Due to the existence of such possibility all machine transients must be studied carefully. "Figure 21" shows the runner strains results of three blades at the pressure and suction side near to the crown connection. All three consecutive curves show the same aspect, fact that is common for machined blades due to the achievement of tight manufacturing tolerances. Also the blades are under the same effect on the pressure and suction sides with higher amplitudes being experienced at the suction blade side. The trend of the stresses is in accordance with the finite element calculation.

There are some excitation frequencies that are going to be present during the machine operation. The goal of the diagnosis is to check if these excitations are going to be amplified by resonance. The wicket gate passing frequency 


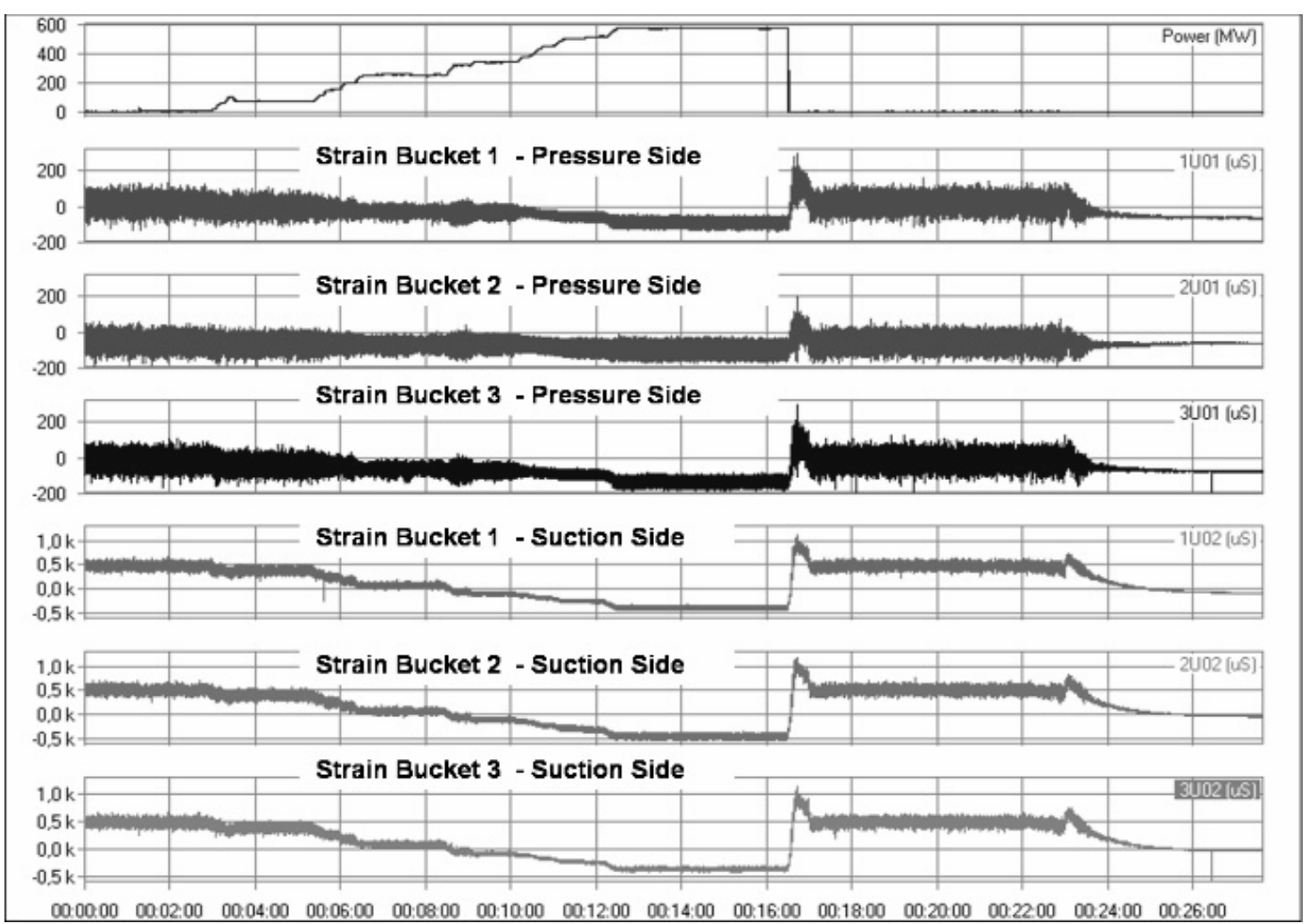

Fig. 21. Strains at 3 consecutive blades $=$ Same behavior Tamdem Cascade - best point.

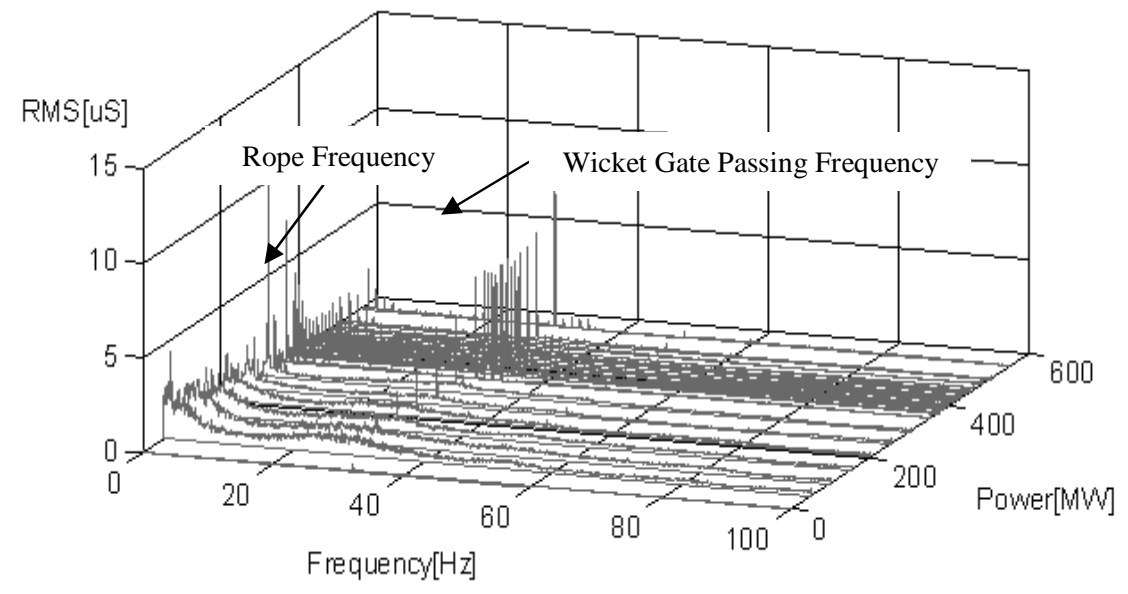

Fig. 22. Water Fall - Discharge edge band.

and harmonics are going to excite the rotating parts. "Figure 22" demonstrates a typical waterfall diagram in the frequency domain where it is possible to recognize the wicket gate exciting frequency growing up as the flow velocity and power increases.

A dynamic stress variation of $3 \%$ up to $8 \%$ of the mean stress portion while the machine is running at the rated operating point is considered within acceptable material limits. Higher values of these amplitudes would indicate amplification and possible damages to the runner material. During part load or low load the stress amplitudes up to 


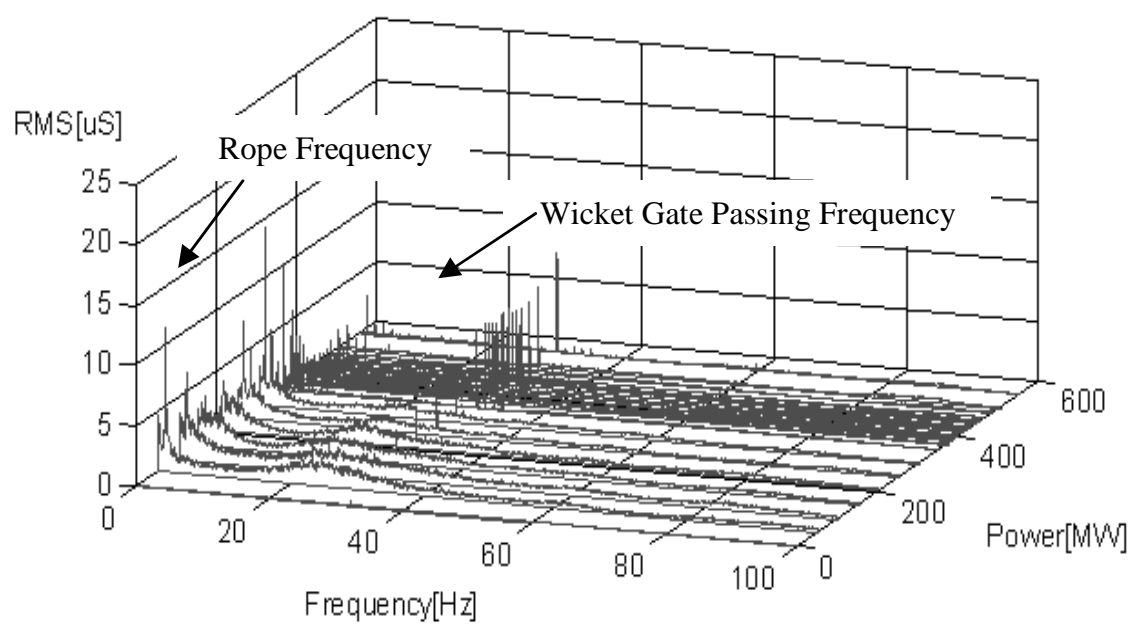

Fig. 23. Water Fall - Discharge Edge Crown.

the wicket gate passing frequency (rotation $\mathrm{x}$ number of wicket gates) should not be higher than $20 \%$ of the mean stress. The machine operating time at this region is limited exactly due to the presence of such instability. Long term operation at this condition will probably cause damages to the material structure of the runner, even for a high strength material such as the ASTM A743 CA6NM series that have yield point above $550 \mathrm{MPa}$ and high toughness typically over $100 \mathrm{~J}$.

High frequency phenomena such as the presence of von Karman trailing edge vortex are commonly over $200 \mathrm{~Hz}$ and might get higher according to machine size and rotation speed. This high frequency excitation, if containing sufficient energy to excite the blades or even to excite a blade natural frequency, can seriously damage the runner causing cracks at the most stressed areas after some months of operation. The occurrence of such phenomena is totally dependent of the runner design and it needs to be avoided in order to enable adequate runner lifetime.

"Figure 24" shows two time series that are related to strain-gage responses that were connected to runner high stress areas. The upper series shows a mean stress of ca. $100 \mathrm{MPa}$ that occurs at $160 \mathrm{MW}$ and the second curve below shows the mean stress values near to $70 \mathrm{MPa}$ occurring at $150 \mathrm{MW}$. These mean values are within the material acceptable limits, however this figure does not permit one to check the frequency and apparently the upper curve represents the critical one for the component lifetime. By checking "Fig. 25", one will see that for the same machine the stresses measured at the same blade location for $130 \mathrm{MW}$ and for $150 \mathrm{MW}$ contain high frequency fluctuation of $200 \mathrm{~Hz}$ with peak to peak amplitude over $40 \%$. The upper curve although showing higher mean stresses does not contain a periodic fluctuation and the stress amplitude is at 5\%, which is acceptable for the runner material. "Figure 26" shows the frequency domain results of several time series from 0 up to $160 \mathrm{MW}$.

This figure clearly shows that the high frequency excitation starts to occur at $110 \mathrm{MW}$ and stops at $160 \mathrm{MW}$. The amplitudes increase from $110 \mathrm{MW}$ up to $150 \mathrm{MW}$ stopping suddenly at $160 \mathrm{MW}$ and this is a phenomena that is dependent from the local flow velocity.

A typical water fall diagram obtained from strain gages that do not feel any high frequency excitation is given on "Fig. 27". This diagram shows the dynamics recorded at runner entrance edges where, in the absence of hydraulic problems, this kind of effect is not percepted inclusively concerning the effects of the rope.

The high frequency occurrence observed at "Fig. 26" might cause a fatigue problem to the runner material. The strain gage related to this figure was placed at the blade band junction, a region that is coincident with welding seams.

Some measures such as local geometry changes, intensity damping methods such as caused by air injection can be tested and can be effective in order to eliminate such an occurrence. It is important to detect the most appropriate method and define under which operating conditions this special feature shall be activated. The diagnosis as proposed here, allied with experienced engineering design data provides these answers. Also a focus must be put on the quality of the welding seam, as well as the mechanical properties of the procedure qualification and especially charpy impact values. 


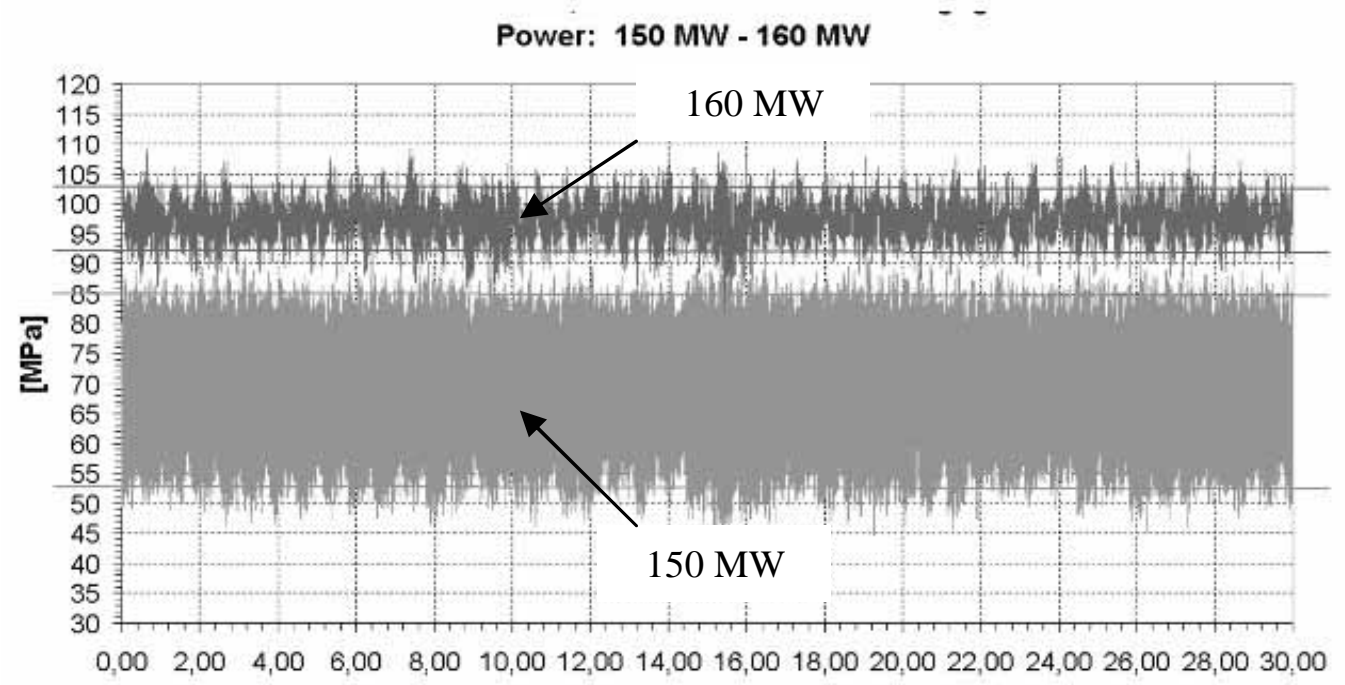

[s]

Fig. 24. High and lower mean stresses.

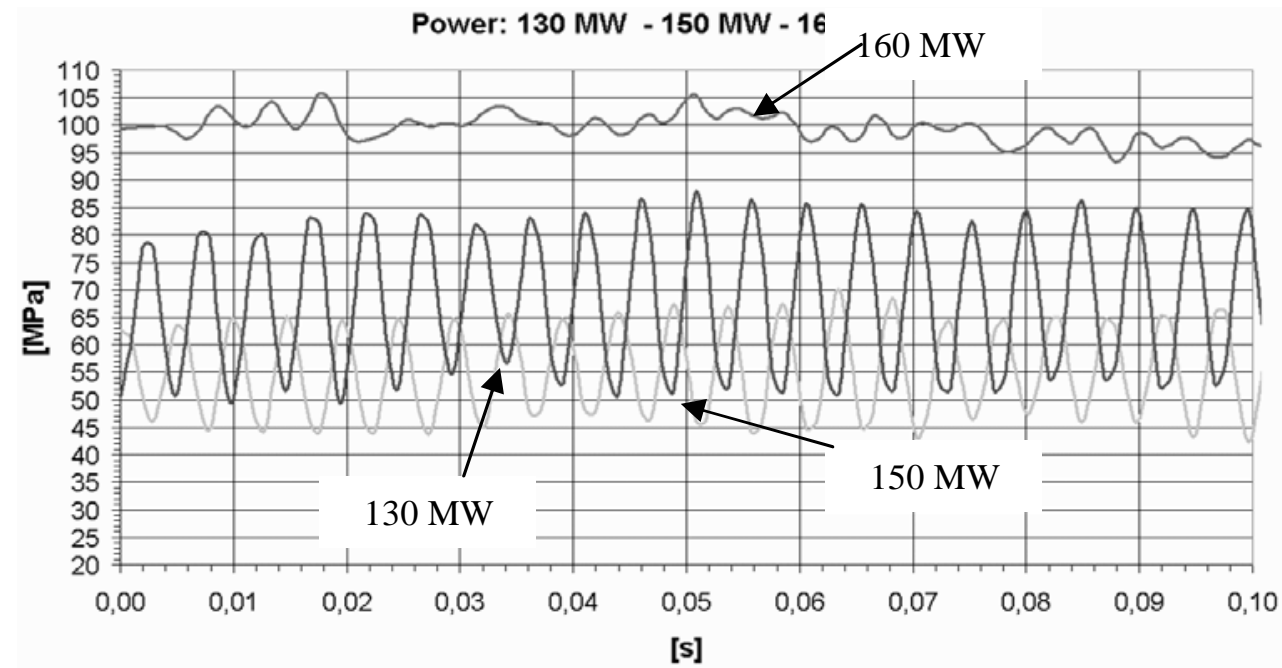

Fig. 25. High frequency amplitude for lower mean stresses.

These interpretation examples summarize briefly the machine most important diagnosis focus. These data when used for fatigue calculations can predict with reasonable accuracy the lifetime of the runner blade and the coupled maintenance cost to repair the damaged areas. Other goals such as the investigation of low and high frequency phenomena on specific operating regions are also commonly added to the diagnosis goals, together with the analysis of machine stability and stress reactions when air is injected through the bottom ring, turbine head cover or admitted though the shaft. 


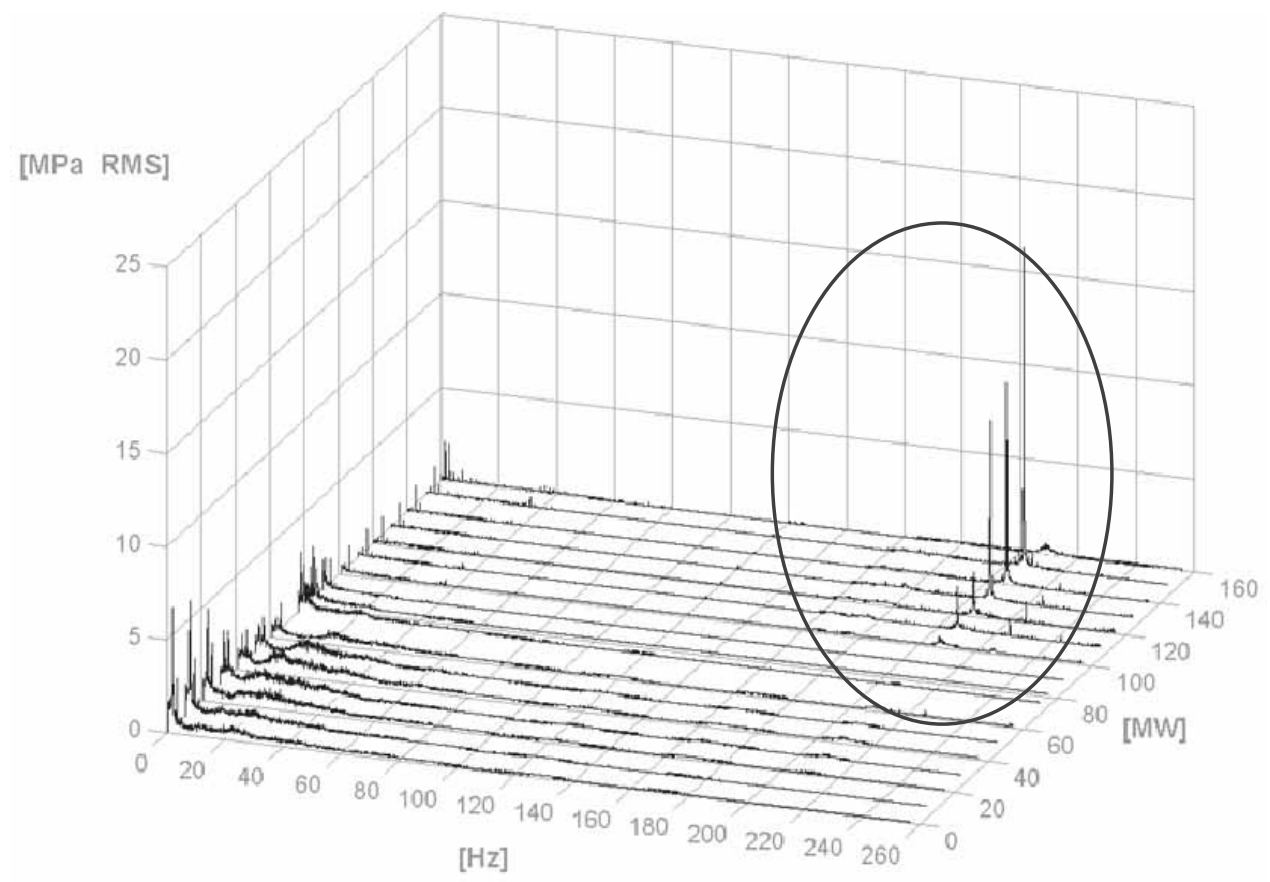

Fig. 26. Water fall of Francis turbine showing high frequency phenomena.

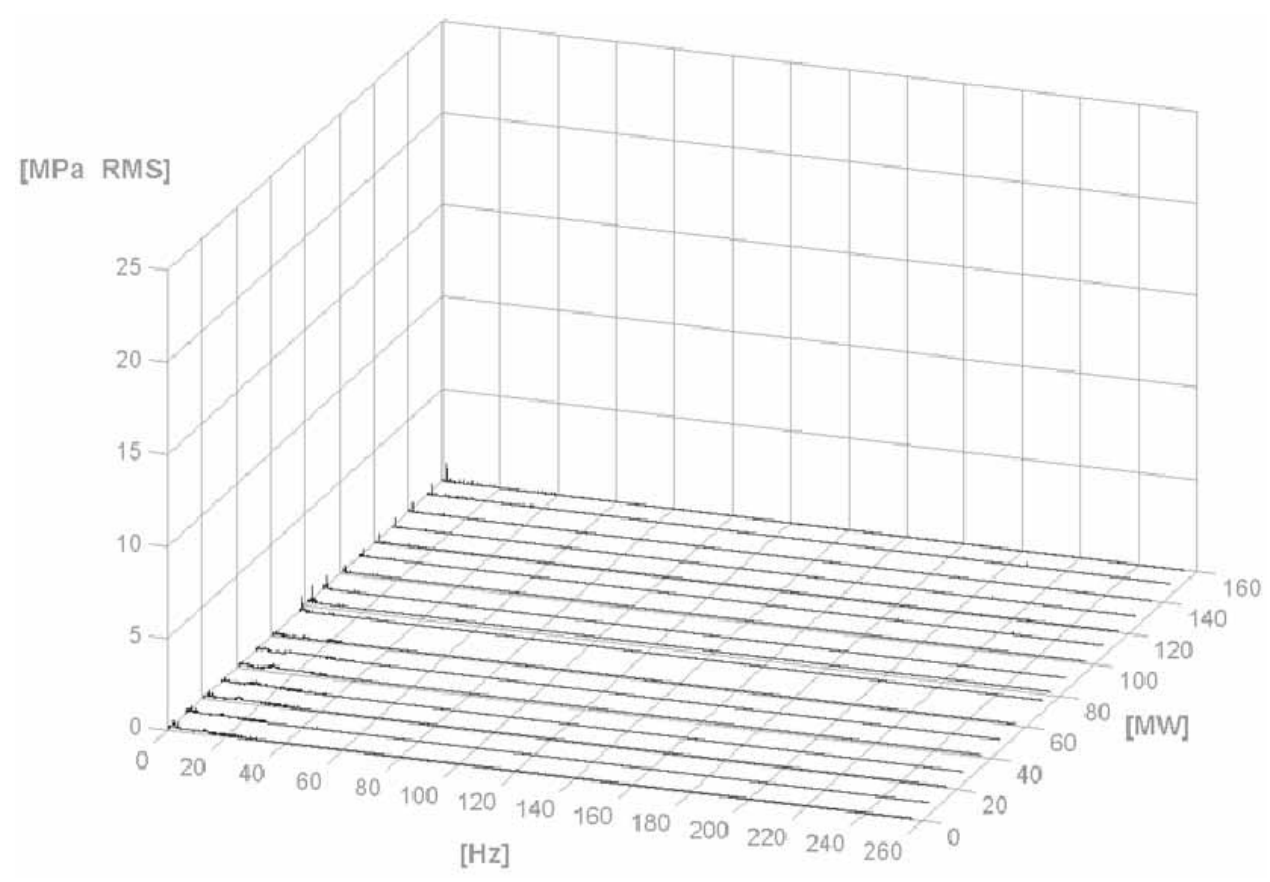

Fig. 27. Water fall of Francis turbine - Aspects without high frequency.

\section{Conclusions \& recommendations}

The acquisition method for a machine diagnosis was described highlighting the set ups and adjustments that must be conducted in order to allow adequate cause-effect correlations. An overview of how a measurement campaign 
must be planned and how it needs to be tailored for a specific Francis machine was demonstrated. Some interpretation examples were shared in order to demonstrate how the data can be used to better understand machine behavior and operational effects since all accumulated experience and knowledge in this specific field is still under high development.

The recommendation for the energy companies is to consider a full machine diagnosis, especially for Francis units, as it is being done frequently in new power plants in the far east, as part of a measure to assure full understanding of hydraulic and structure mechanical behavior and to tailor the unit operating mode by the use of this acquired knowledge in order to optimize the return of investment and keep the maintenance under comfortable limits.

\section{Responsibility notice}

The first author is the only responsible for the data included in this paper. All presented data are accumulated from several experiences and do not correspond to any specific machine. The only aim of this paper is to illustrate the method and corresponding interpretation.

\section{References}

[1] R.K. Fisher, U. Seidel, G. Grosse, W. Gfeller and R. Klinger, Case Study in Resonant Hydroelastic Vibration: The Causes of Runner Cracks and the Solutions Implemented for the Xiaolangdi Hydroelectric Project, Proceedings of XXIst IAHR Symposium - Section of Hydraulic Machinery and Systems, Vol. II, Lausanne, Switzerland, 2002, 895-906.

[2] P.Y. Lowys, F. Paquet, P.Y. Lowys, M. Couston, M. Farhat, S. Natal and F. Avellan, On Board Measurements of Pressure and Strain Fluctuation in a Model of Low Head Francis Turbines Part 2: Measurements and Preliminary Analysis Results, Proceedings of XXIst IAHR Symposium - Section of Hydraulic Machinery and Systems, Vol. II, Lausanne, Switzerland, 2002, 865-872.

[3] L.T.L. Freitas, J.C. Zanutto and H.G. Poll, Comportamento Dinâmico de rotores Francis de Baixa Queda, Proceedings of the 2nd Power Plant Seminar on Modernization and Rehabitiltation, CD, São Paulo, Brazil, 2001.

[4] P. Ebersbach and H. Irretier, On the application of modal parameter estimation using frequency domain algorithms, in: 6th International Modal Analysis Conference, Kissimmee, Florida, feb., 1988. Proceedings. Union College, Shenectady, NY., Society of Experimental Mechanics, Inc., Bethel, CT, v.1, 1988, 113-120.

[5] H. Irretier and F. Reuter, Experimentelle Modalanalyse in rotierenden Maschinen - Abschlussbericht, Institut fir Mechanik, Universität Gesamthochschule Kassel, Kassel, Mai, 1994.

[6] M. Dubas, Über die Erregung infolge der Periodiziät von Turbomaschinen, Ingenieur-Archiv 54 (1984), $413-426$.

[7] S.J. Wildheim, Excitation of Rotating Circunferentially Periodic Structures, Journal of Sound and Vibration 75(3) (1981), $397-416$.

[8] W.J.A. Ponge-Ferreira, F.E.B. Nigro and H. Irretier, Identifying Resonances in Rotating Structures with Interference Diagrams, IFToMM 6th Int. Conf. on Rotor Dynamics, Sydney, Australia, Oct. 2, 2002.

[9] D.J. Mead, Wave propagation in continuos periodic structures: research contributions form Southampton, 1964-1995, Journal of Sound \& Vibr 190(3) (Feb. 29, 1996), 495-524.

[10] W.J.A. Ponge-Ferreira, H. Irretier and F.E.B. Nigro, Interference Diagrams for the Characterization of Resonances in Rotating Structures, EURODINAME99 - International Symposium on Dynamic Problems in Mechanics and Mechatronics, University of Ulm, Proceedings, July 1999, 45-51.

[11] W.J.A. Ponge-Ferreira and F.E.B. Nigro, Método de Identificação de Ressonâncias em Estruturas Periódicas Cíclicas em Rotação. Escola Politécnica da Universidade de São Paulo. São Paulo, junho, 2000. (Tese de Doutorado).

[12] C.A. Gongwer, A study of vanes singing in water. Transactions of the ASME, Journal of Applied Mechanics, Paper 52-APM-33, pp. 432438, Dec. 1952. / Apresentado a National Conference of the Applied Mechanics Division of the ASME, State College, 1952.

[13] Y.N. Chen and D. Florjancic, Karman-vortex-induced vibrations on guide vanes with slender trailing edges, Conference on vibrations and noise in pump, fan and compressor installations, Southampton, 1975. Proceedings. [s.n.]. 69-77.

[14] C. Liess, Flow induced stress fluctuations in stay vanes of large hydraulic turbines, International conference on flow induced vibrations, Bowness-on-Windermere, 1987. Proceedings. [s.n.]. 157-170.

[15] K. Miyagawa, S. Fukao and Y. Kawata, Study on stay vane instability due to vortex shedding, IAHR SYMPOSIUM, 22., Stockholm, 2004. Proceedings. Paper B12-2. 1 CD-ROM. 

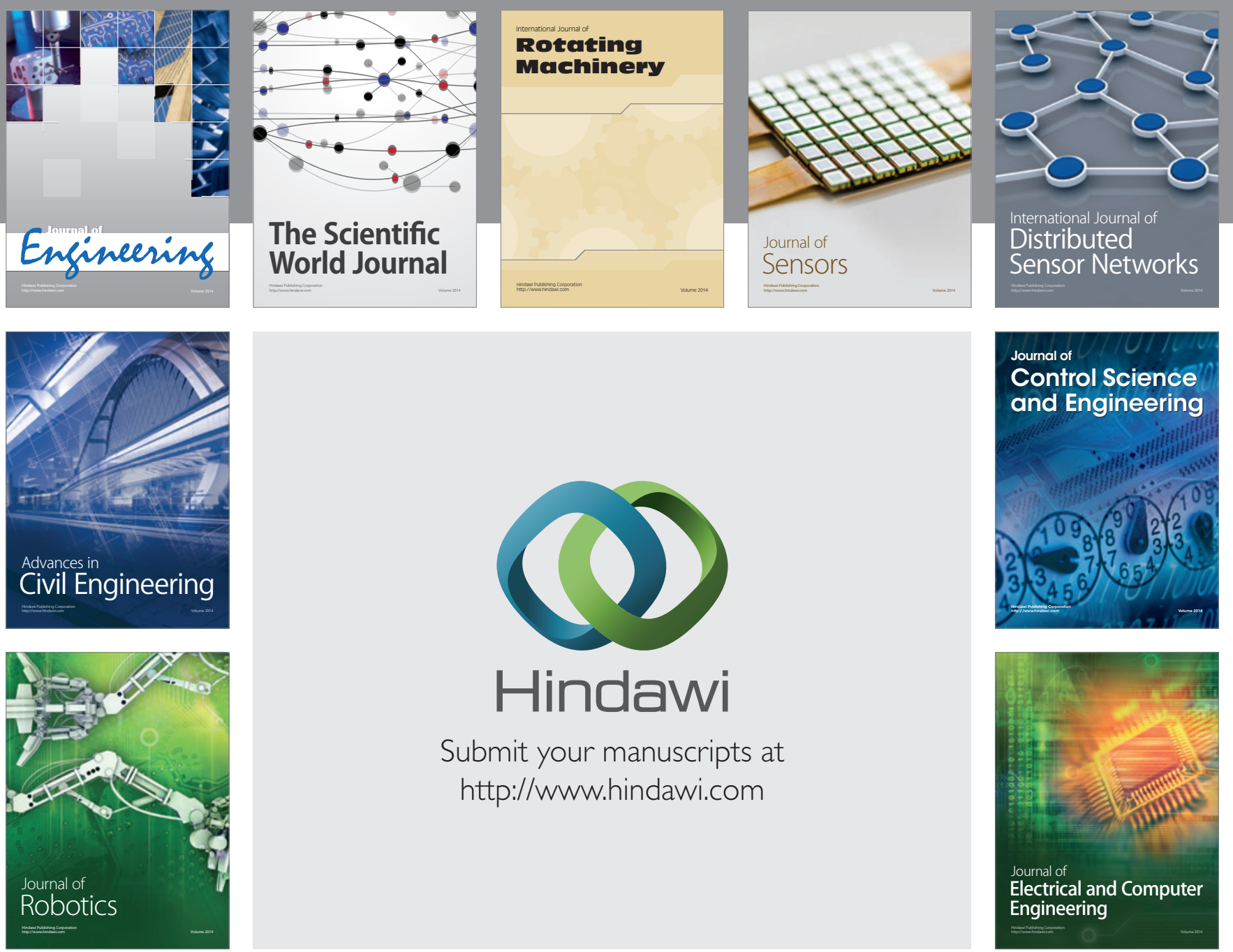

Submit your manuscripts at

http://www.hindawi.com
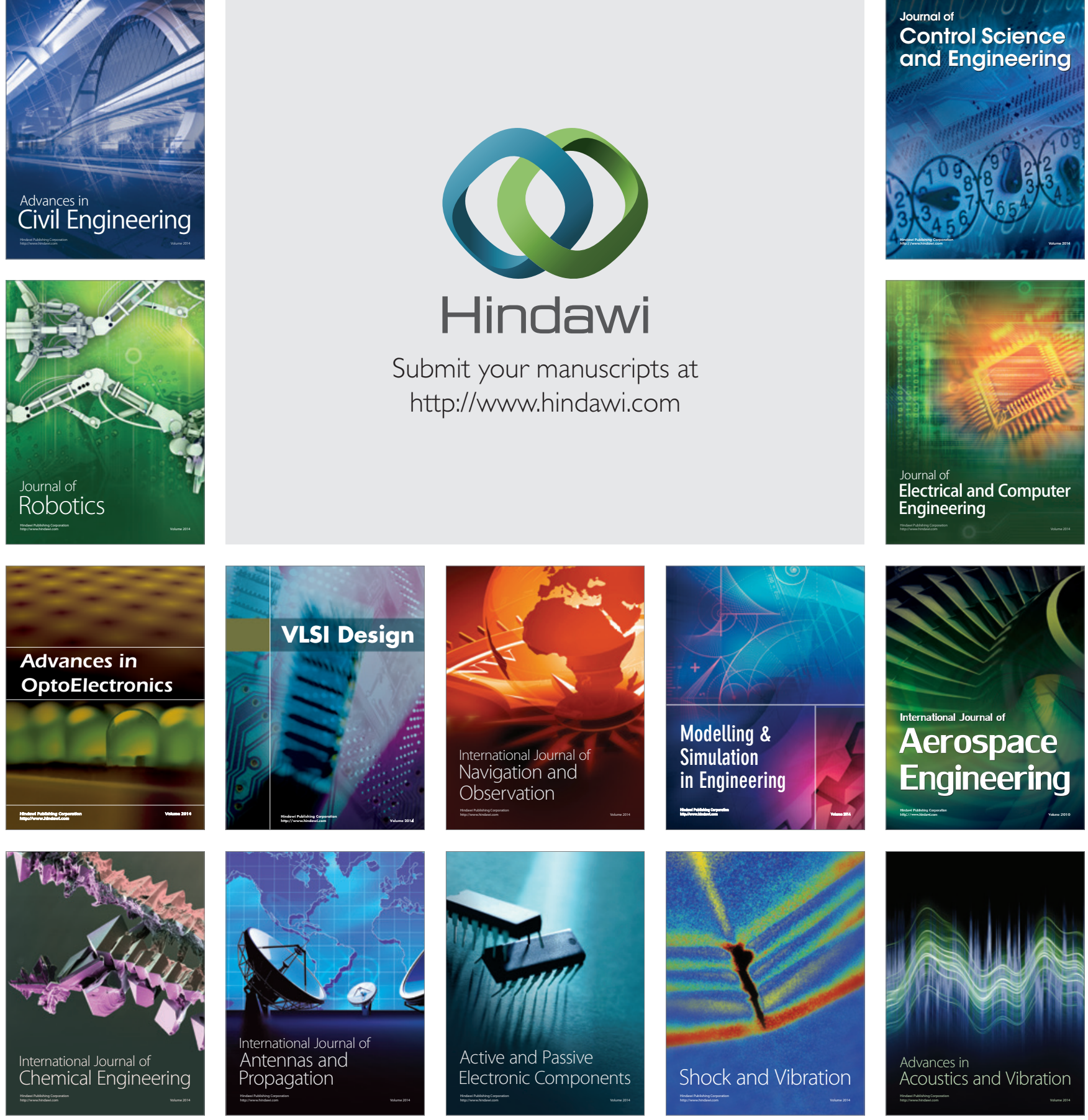